\title{
Electromagnetic modelling of three-dimensional metallic photonic crystals
}

\author{
G. Guida, D. Maystre, G. Tayeb and P. Vincent \\ Laboratoire d'Optique Electromagnétique \\ Faculté des Sciences et Techniques de St-Jérôme, case 262 \\ 13397 Marseille CEDEX 20, France
}

\begin{abstract}
In order to investigate the properties of threedimensional metallic photonic crystals, we have developed an adaptation of Harrington's method for scattering by thin wires. Due to the peculiarity of photonic crystals, we have been led to renounce to two assumptions: the current intensity in the wires is not assumed to vanish at a free extremity of a wire and the Kirchhoff law at a junction between wires is not used any longer. It is shown that, paradoxically, the numerical implementation is simplified by these changes and that our method provides a better convergence of the results. Using our code, the properties of 3D metallic photonic crystals are investigated and compared with those of 2D crystals.
\end{abstract}

\section{Introduction}

The last few years have seen a growing interest in the theoretical and experimental studies of photonic band structures [1-8]. The basic feature of these structures is to provide a complete control of light propagation. Dielectric photonic crystals are able to inhibit spontaneous emission of light and could permit the construction of zero threshold lasers and single mode light emitting diodes.

In this paper, we are dealing with another kind of photonic band structure: the metallic photonic crystal. It is well known that dielectric photonic crystals present gaps limited to one octave or even less (see for instance $[3,6,8,9]$ for some examples of such gaps for doped or non-doped crystals). In contrast, metallic photonic crystals generate gaps extending from the null frequency to a cut-off value. Another big difference is that metallic crystals are mainly intended to work in the microwaves region, for instance as efficient reflectors or resonant cavities (see [10-16] for more details), and dielectric photonic crystals are expected to work in the optical region.

The metallic crystal we are concerned with in this paper is made by a three-dimensional (3D) finite periodic array of perfectly conducting wires. These wires are parallel to the three axes of a cartesian coordinates system and the elementary cell is a cube, but the computer code we have made could investigate the properties of more complicated metallic photonic crystals, presenting other kinds of symmetries. The theory we have used is based on the Electric Field Integral Equation (EFIE) proposed by Harrington for wire antennas [17]. Indeed, the wires radius is assumed to be very small compared with the wires length or with the wavelength of the incident field. A Galerkin method with piecewise linear (triangular) symmetric basis and test functions is used. Classically, it is assumed that the current vanishes at a free extremity of a wire and thus the basis functions are null on this extremity. In this paper, we explain why this assumption is questionable, at least when the length of a wire is smaller than half a wavelength. Hence, we renounce to this assumption and we add to the symmetrical triangular finite elements a saw-tooth function with discontinuity at the end of the wire. In the same way, it is no more assumed that Kirchhoff's law is satisfied at a junction between wires [18] and similarly, a saw-tooth function is used at an extremity of a wire, with discontinuity on the junction. It is quite interesting to notice a paradoxical result: the numerical implementation is simpler after renouncing to these two hypotheses. The computer code is carefully checked using many classical tests and we verify that the current can significantly differ from zero at the ends of a wire.

It is shown that the transmission gap of the structure starts at zero frequency. Moreover, comparing the 
numerical results with those obtained from computer codes dealing with two-dimensional (2D) metallic photonic crystals $[8,19]$ lead us to a very important conclusion: the two-dimensional model, much simpler to handle, is able to bring vital informations on the properties of these structures. As a consequence, the conjecture according to which a metallic photonic crystal can simulate a homogeneous material with a plasmon frequency in the microwaves region [20], already shown for 2D crystals [21], should extend to 3D metallic crystals.

\section{Basic equation}

Throughout the paper, we use the complex notation, assuming a time dependence in $\exp (-i \omega t)$. An incident plane wave with electric field $\vec{E}^{i}$ of wavelength $\lambda=2 \pi / k$ propagating in vacuum illuminates a system of $W$ perfectly conducting wires with circular crosssection of radius $r \ll \lambda$.

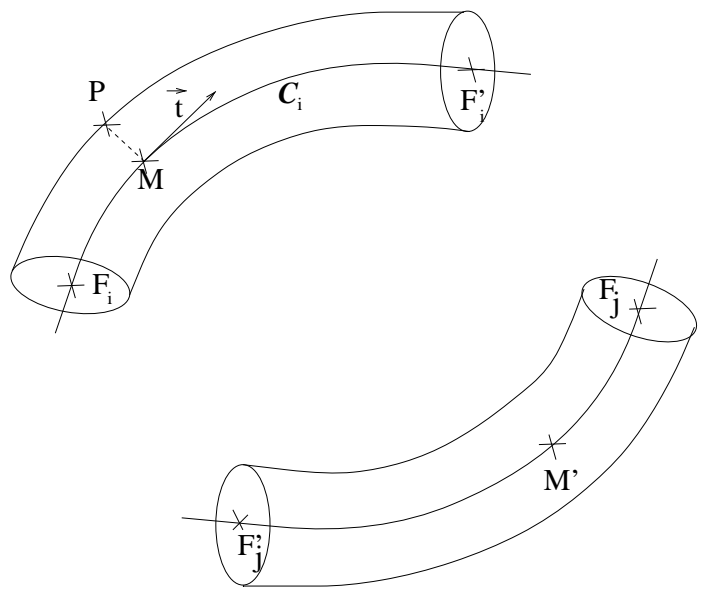

Figure 1: Notations.

We define (Fig. 1) the extremities $F_{i}$ and $F_{i}^{\prime}(i \in$ $[1, W]$ ) of each wire (the choice of the extremity called $F_{i}$ can be made arbitrarily). A junction will always be considered as an extremity of each of the connected wires. The axis $\mathcal{C}_{i}$ of each wire is oriented from $F_{i}$ to $F_{i}^{\prime}$ and $\vec{t}(M)$ is the unit tangent vector at a point $M$ of $\mathcal{C}, \mathcal{C}$ being defined as the set of $\mathcal{C}_{i}$. As in the work of Harrington [17], we assume that the current can be approximated by filaments of current $I\left(M^{\prime}\right)$ on $\mathcal{C}, I\left(M^{\prime}\right)$ taking algebraic values deduced from the orientation of $\mathcal{C}$ at point $M^{\prime}$. In the same way, the boundary condi- tion (the tangential value of the total field $\vec{E}(P)$ must vanish at a point $P$ placed at the surface of the metal) is applied only to the axial component of $\vec{E}(P)$ at the wire surface. Assuming that $M$ is the point of the wire axis in the same cross-section as $P$, the incident field $\vec{E}^{i}(P)$ is approximated by its value on the axis $\vec{E}^{i}(M)$, and $\left|\overrightarrow{P M}^{\prime}\right|$ by [17]:

$$
\left|\overrightarrow{P M}^{\prime}\right| \approx R=\sqrt{M M^{\prime 2}+r^{2}}
$$

The basic equations given by Harrington yield:

$$
\begin{array}{r}
\frac{1}{i \omega \epsilon_{0}} \int_{\mathcal{C}} \frac{d I}{d M^{\prime}}\left(M^{\prime}\right) \frac{d G}{d M}\left(M, M^{\prime}\right) d M^{\prime} \\
-i \omega \mu_{0} \int_{\mathcal{C}}\left[\vec{t}(M) \cdot \vec{t}\left(M^{\prime}\right)\right] I\left(M^{\prime}\right) G\left(M, M^{\prime}\right) d M^{\prime} \\
=\vec{t}(M) \cdot \vec{E}^{i}(M),
\end{array}
$$

where:

$$
G\left(M, M^{\prime}\right)=\frac{\exp (i k R)}{4 \pi R}
$$

\section{About some assumptions on the current intensity}

In the theory of Harrington [17], the following assumption is made: "It should be noted that the end point of a wire is treated as the center of an interval with zero current. This is suggested in Fig. 4.1.b by starting the intervals one-half subsection in from the wire ends. It is mathematically equivalent to the boundary condition $I=0$ at the ends of a wire".

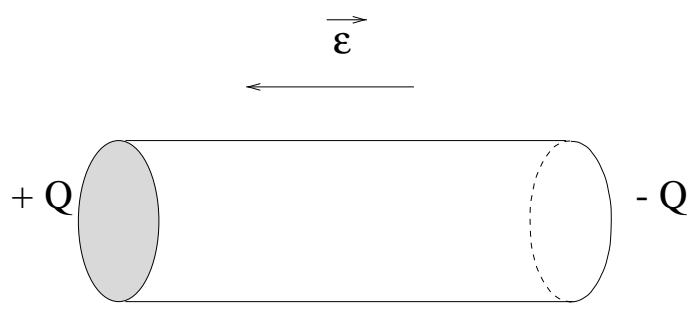

Figure 2: About the assumption of an intensity equal to zero at an extremity of a wire. Charges $+Q$ and $-Q$ are created at the extremities by the static electric field $\overrightarrow{\mathcal{E}}$.

We do not adopt this assumption. Indeed, let us consider the wire represented in Fig. 2, in a static electric field $\overrightarrow{\mathcal{E}}$ (of course the complex notation is abandoned in 
this example) parallel to the axis of the wire. We assume that this wire is not connected to other ones. In order to establish a total field which vanishes inside the wire, electric charges $+Q$ and $-Q$ will be created on both extremities of the wire. These charges will generate inside the wire an electric field $-\overrightarrow{\mathcal{E}}$.

Now, let us replace the static field $\overrightarrow{\mathcal{E}}$ by an harmonic field $\overrightarrow{\mathcal{E}} \cos (\omega t)$. In the low frequency domain, one can consider that the charge $Q\left(t_{0}\right)$ generated at $t=t_{0}$ on the extremity of the wire will be equal to the charge which would be generated by a static electric field $\overrightarrow{\mathcal{E}} \cos \left(\omega t_{0}\right)$. The fact that the charge $Q(t)$ is not constant implies that the current intensity at both extremities of the wires does not vanish, and this current is an increasing function of the section of the wire.

Of course, this reasoning is made in the quasi-static regime, where the current is constant between the extremities of the wire. Even though this remark does not hold in high frequency, it is not clear that the intensity at extremities is negligible. In the same way, we do not assume the conservation of intensity at a junction between wires since the charge on the junction may vary with time.

At a first glance, one could think that renouncing to these assumptions complicates the numerical implementation. Paradoxically, it is not the case. Indeed, it must be observed that many authors have been led to introduce continuations of the wires at the extremities, or at the junctions $[22,23]$. If these continuations are suppressed, the system of equations to be solved does not constitute a square system. It will be shown that this difficulty completely disappears in our numerical implementation.

\section{Numerical implementation}

First, let us come back to the approximation of $P M^{\prime}$ made in Eq. (1). It is obvious that this approximation is convenient for a precise calculation of $G\left(M, M^{\prime}\right)$ when $M M^{\prime} \gg r$. When $M M^{\prime}$ and $r$ have the same order of magnitude, this approximation remains very good provided that $M M^{\prime}$ and $M P$ are nearly orthogonal. This condition is filled when $M$ and $M^{\prime}$ are located on the same wire, the radius of curvature of this wire being much greater than $r$. On the other hand, this approximation may make problem when $M$ and $M^{\prime}$ are located on two different wires. However, Eq. (1) has been used in that case as well. The main reason is that a more precise calculation of $P M^{\prime}$ would lead to considerable complications. The second one is that, in that case, even the basic approximation introduced in the theory (the intensity in the wire is replaced by a filament of current) can be questioned. This remark explains why we have performed a thorough validation of our numerical results.

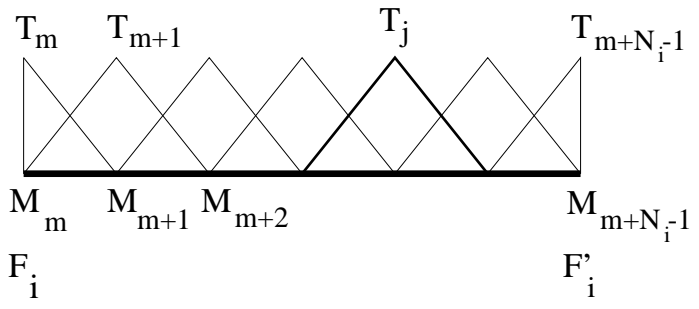

Figure 3: Triangular finite elements on the $i^{t h}$ wire.

In order to implement Eq. (2) on the computer, the intensity is projected on finite elements $T_{m}$ defined in Fig. 3, where, for simplicity, the wire is drawn as a linear segment. On each wire, a set of $N_{i}$ equally spaced points $M_{j}$ is defined. The index $j$ is running continuously from the first point $F_{1}$ of the first wire to the last one $F_{W}^{\prime}$ of the $W$-th wire. This set of points allows us to define a set of triangular finite elements $T_{j}$ represented in Fig. 3. In contrast with the other finite elements, which are symmetrical, the finite elements placed at the extremities of a segment have a saw-tooth shape. The finite element $T_{m}$ decreases linearly from a unit value at $M_{m} \equiv F_{i}$ to a zero value at $M_{m+1}$, while $T_{m+N_{i}-1}$ increases from zero at $M_{m+N_{i}-2}$ to 1 at $M_{m+N_{i}-1}$. It is worth noticing that a connection point between $q$ wires is represented by $q$ extremities of finite elements. Hence, the total number of finite elements is denoted by $N_{t o t}$. The intensity is now given by:

$$
I(M)=\sum_{m=1}^{N_{t o t}} I_{m} T_{m}(M)
$$

It is crucial to notice that no condition is enforced on the values of $I_{m}$ corresponding to points $M_{m}$ located at the extremities of the wires since we do not assume that the intensity vanishes at a free extremity of a wire, or that the intensity is conserved at a connecting point.

Using a Galerkin method, Eq. (2) is projected on the same set of finite elements and we get $\forall n \in\left[1, N_{t o t}\right]$ :

$$
\frac{1}{i \omega \epsilon_{0}} \sum_{m=1}^{N_{t o t}} I_{m} \int_{\mathcal{C}} \int_{\mathcal{C}} T_{n}(M) T_{m}^{\prime}\left(M^{\prime}\right)
$$




$$
\begin{array}{r}
\frac{d G}{d M}\left(M, M^{\prime}\right) d M^{\prime} d M \\
-i \omega \mu_{0} \sum_{m=1}^{N_{\text {tot }}} I_{m} \int_{\mathcal{C}} \int_{\mathcal{C}} \vec{t}(M) \cdot \vec{t}\left(M^{\prime}\right) T_{n}(M) T_{m}\left(M^{\prime}\right) \\
G\left(M, M^{\prime}\right) d M^{\prime} d M \\
=\int_{\mathcal{C}} \vec{t}(M) \cdot \vec{E}^{i}(M)
\end{array}
$$

where $T_{m}^{\prime}\left(M^{\prime}\right)$ stands for the derivative $d T_{m}\left(M^{\prime}\right) / d M^{\prime}$. By integrating by parts the first term of the left-hand side of (5), we deduce finally a linear set of $N_{t o t}$ equations with $N_{t o t}$ unknowns $I_{m}$ :

$$
\forall n \in\left[1, N_{t o t}\right], \quad \sum_{m=1}^{N_{\text {tot }}} A_{n, m} I_{m}=S_{n}
$$

with:

$$
\begin{gathered}
A_{n, m}=\frac{-1}{i \omega \epsilon_{0}} V_{n, m}-i \omega \mu_{0} W_{n, m} \\
V_{n, m}=\int_{\mathcal{C}} \int_{\mathcal{C}} T_{n}^{\prime}(M) T_{m}^{\prime}\left(M^{\prime}\right) G\left(M, M^{\prime}\right) d M^{\prime} d M
\end{gathered}
$$

$$
\begin{array}{r}
W_{n, m}=\int_{\mathcal{C}} \int_{\mathcal{C}} \vec{t}(M) \cdot \vec{t}\left(M^{\prime}\right) T_{n}(M) T_{m}\left(M^{\prime}\right) \\
G\left(M, M^{\prime}\right) d M^{\prime} d M \\
S_{n}=\int_{\mathcal{C}} \vec{t}(M) \cdot \vec{E}^{i}(M) \quad T_{n}(M) d M
\end{array}
$$

At a first glance, the expression of $V_{n, m}$ given by Eq. (8) makes problems since the derivative $T_{m}^{\prime}$ is not defined (in the sense of functions) at points $M_{m}$ located at an extremity of a wire, due to the saw-tooth shape of the finite elements on these points. A possible answer to this problem is to consider $T^{\prime}$ as a distribution including a Dirac distribution. In practice, it is much simpler to interpret the definition of these finite elements in a new way. We consider that the finite element $T_{m}$ associated with the first extremity of a wire is defined as the limit value when $u \rightarrow 0$ of the finite element represented in Fig. 4.

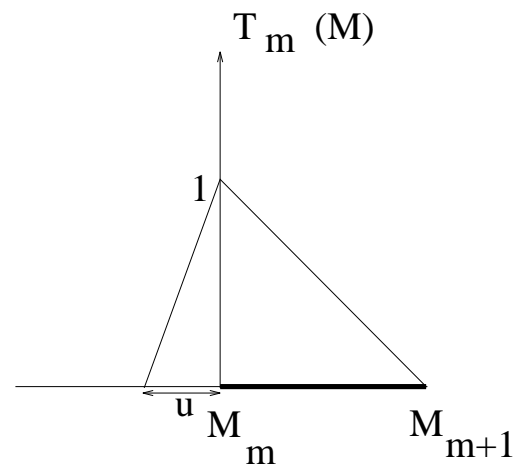

Figure 4: Representation of a finite element placed at the first extremity of a wire taking into account the possible non-zero value of the intensity at the end of the wire.

A symmetrical interpretation is given to the finite element associated with the second extremity of a wire. The integrals in the right-hand member of Eqs. (8), (9) and (10) are made by using a small value of $u$. Of course, it has been verified that the $I_{m}$ tend to a limit value as $u$ tends to zero. In practice, it suffices to take for $u$ a value less than $10^{-3}$ times the width of a symmetrical finite element.

The double integrals are achieved numerically by using an adaptive algorithm proposed by Berntsen et al [24], [25] to avoid any a priori approximation. This algorithm permits a numerical integration over hyperrectangular regions of multiple integrals by means of a globally adaptive subdivision strategy. The reliability of the integration is improved by a careful evaluation of the error at each step of the algorithm and in each dimension, the user can choose among several integration rules. For our calculations, we have chosen an integration rule of polynomial degree 7 . This rule gives a rapid convergence for such double integrals.

\section{Validation of the theory}

We have checked the validity of the results for two kind$\mathrm{s}$ of scattering objects. The first one is a rectilinear wire of finite length and the second one a system of three connected wires. The first test was a test of convergence of the results when the number $N_{t o t}$ of finite elements is increased. The length of the single wire was fixed to $0.3 \lambda$ and its radius to $10^{-2} \lambda$. The incident wave propagates in a direction perpendicular to the wire, with an 
electric field parallel to the wire.

Figure 5 shows the modulus of the intensity for 4 values of $N_{\lambda}$, the number of points per wavelength. The curves obtained for $10 \leq N_{\lambda} \leq 50$ are nearly identical. When $N_{\lambda}$ exceeds the value of 50, oscillations begin to appear at both extremities of the wire. These oscillations become stronger and stronger when $N_{\lambda}$ is increased (Fig. 5b). In our opinion, the origin of the divergence for large values of $N_{\lambda}$, already found by other authors [18], must be found in the basic approximation: the current intensity cannot be approximated by a filament of current located on the wire axis any longer. Indeed, the calculation of the double integrals in the right-hand members of (8) and (9) can be interpreted as the calculation of the interaction between current intensities located on the ranges of finite elements $T_{n}$ and $T_{m}$. If these ranges are very close to each other (for instance if they are neighbors) and if the width of the finite elements have the same order of magnitude as the diameter of the wire, obviously this basic approximation fails: a calculation of matrix elements $V_{n, m}$ and $W_{n, m}$ made by translating one of the filaments of current towards the surface of the wire would provide very different results. This remark inclines us to think that the phenomenon of divergence of the results for large values of $N_{\lambda}$ is strengthened when $r / \lambda$ increases.
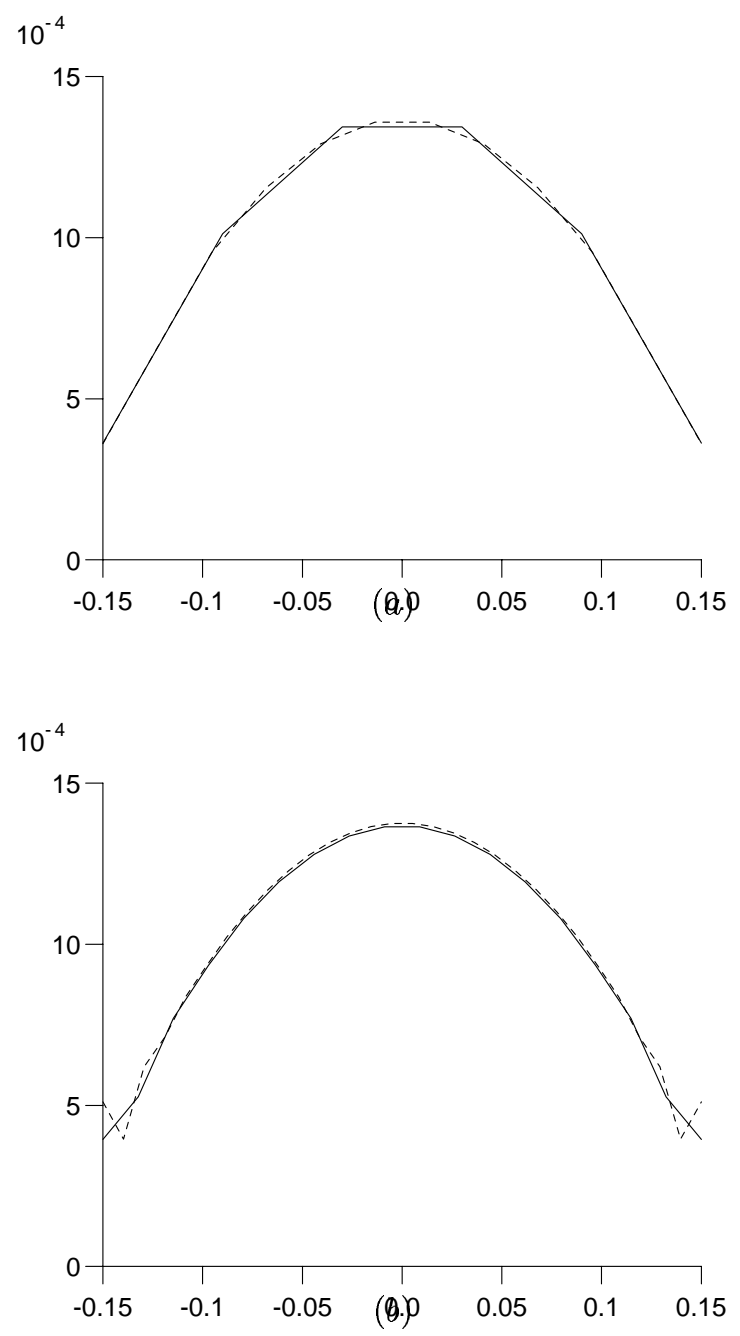

Figure 5: Convergence of the modulus of the current along a single wire of length $0.3 \lambda$ and radius $r=10^{-2} \lambda$ for various values of the number of elements per wavelength $N_{\lambda}$ : a) $N_{\lambda}=20$ (solid curve) and $N_{\lambda}=40$ (dashed curve); b) $N_{\lambda}=60$ (solid curve) and $N_{\lambda}=$ 100 (dashed curve).

This conjecture has been confirmed by our numerical results. For a radius $r=10^{-2} \lambda$, the limit $\left(N_{\lambda}=50\right)$ was reached for a distance $d$ between two discretization points equal to $\lambda / 50$, value of the diameter of the wire. If we adopt this rule of thumb, and recalling that 10 points of discretization at least per wavelength are necessary, we can conjecture that the method will be 
unable to deal with wire diameters larger than $\lambda / 10$. Thus we should restrict our calculations to wires such that $r<\lambda / 20$. This prediction was fully confirmed by the reciprocity [26] and energy balance criteria [27]. These criteria are satisfied to within $1 \%$ in the range of $r / \lambda$ specified previously, provided that the requirements $N_{\lambda} \geq 10$ and $2 r<d$ are satisfied.

A vital remark must be made looking at Fig. 5a: the intensities on both sides of the wire are not negligible. On the other hand, it can be seen in

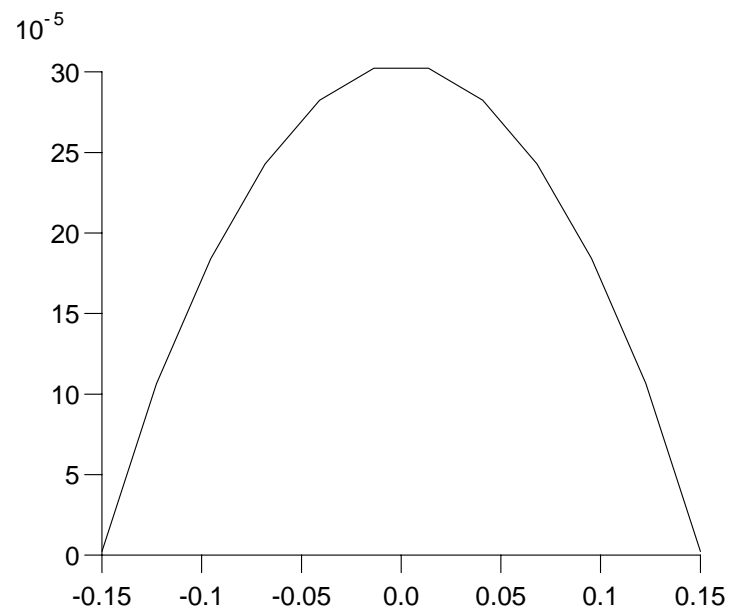

Figure 6: The same as Fig. 5 (a), but for $N_{\lambda}=40$ and $r=10^{-4} \lambda$.

Fig. 6 that the relative value of the intensity on both sides of the wire (compared with the intensity at the middle) tends to zero when $r / \lambda$ decreases, accordingly to the remark about the quasi-static regime made in section 3. The same conclusion holds when the length of the wire is increased: the relative value of the intensity on both sides decreases when the length of the wire exceeds $\lambda / 2$ (resonant antenna), even though the absolute value of these intensities keeps the same order of magnitude. This explains why the current at both extremities can be neglected in most cases of antennas. In the present paper, we are not concerned with antennas but with photonic crystals, and we will see that the wires can have lengths smaller than half a wavelength, thus the currents at extremities of the wires cannot be neglected in general.

We found it interesting to check the importance of the classical assumption (the current vanishes at both extremities of the wire) for the scattered field or for the value of the current at other points. With this aim, we represent in Figs. 7 and 8 the current intensity and the bistatic cross section of the wires considered in Fig. 5 and 6.

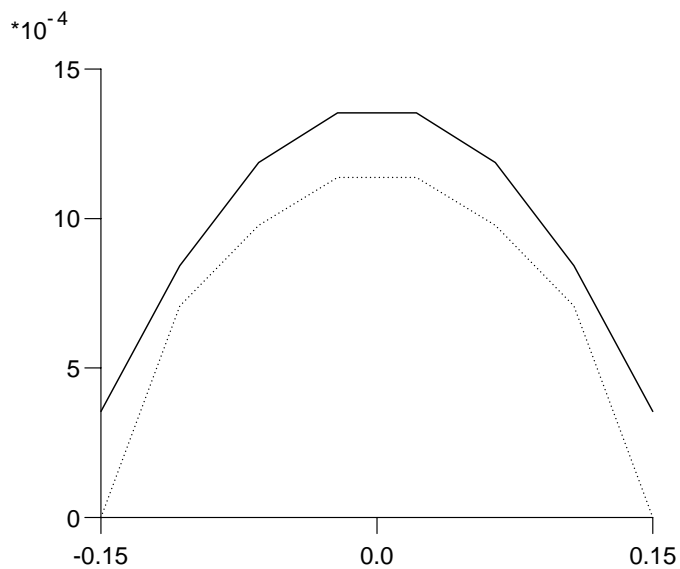

(a)

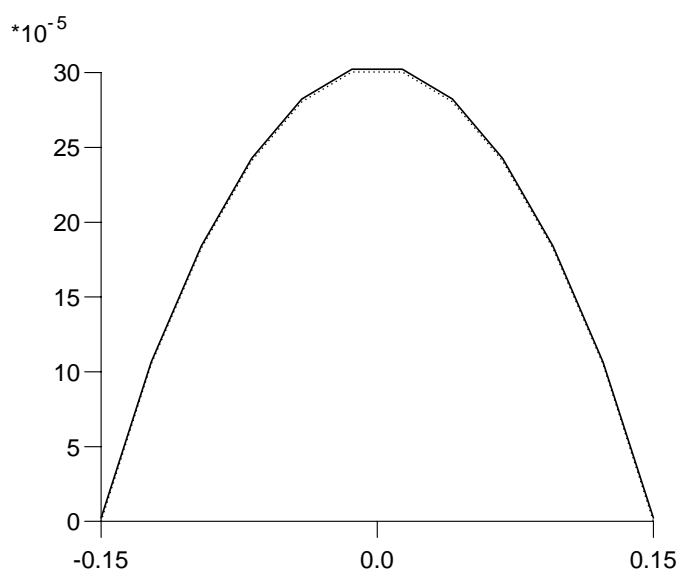

(b)

Figure 7: Modulus of the current along a single wire of length $0.3 \lambda$ and radius (a): $r=10^{-2} \lambda$ or (b): $r=$ $10^{-4} \lambda$. The solid curve is obtained from our method and the dotted curve by assuming the current intensity to vanish at both extremities.

In these figures, the solid curve is obtained from our 
method whereas the dotted line represents the results obtained by eliminating the two saw-tooth finite elements at the extremities of the wire. With this change, we impose the current intensity to vanish at both extremities.
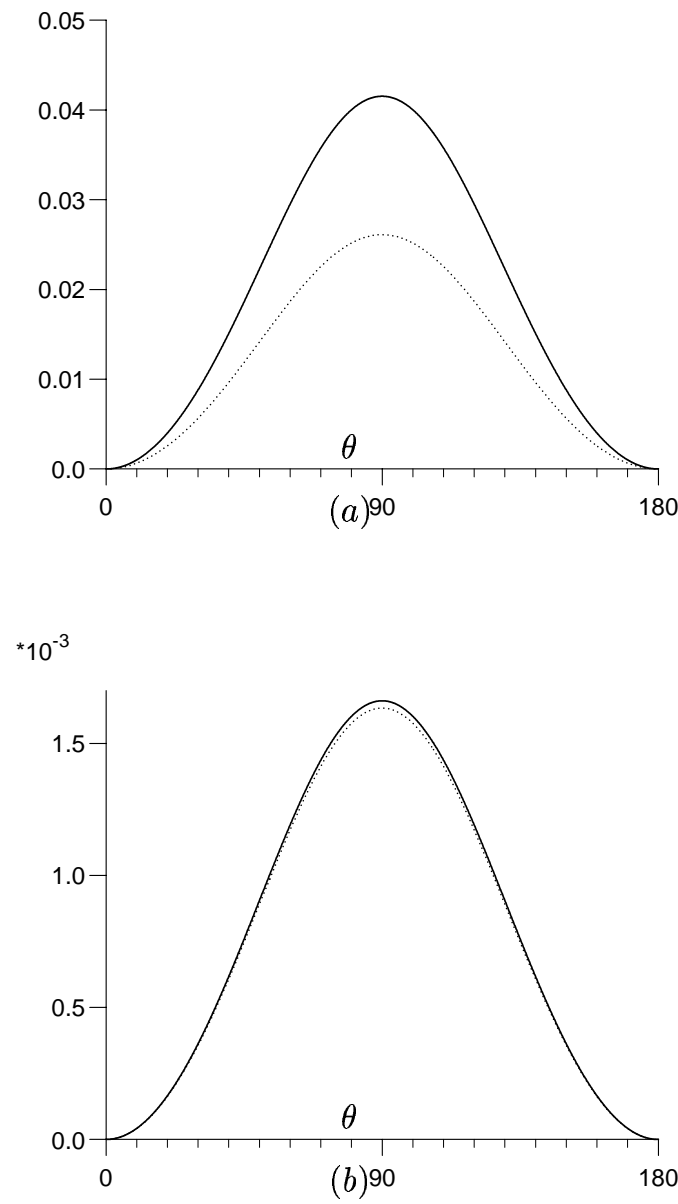

Figure 8: The same as figure 7, but for the bistatic cross section.

In Fig. 8, $\theta$ denotes the angle between the wire and the direction of observation. Since the theory assumes that the current in the wire is replaced by a filament placed on the axis, the scattered intensities depend on $\theta$ only.

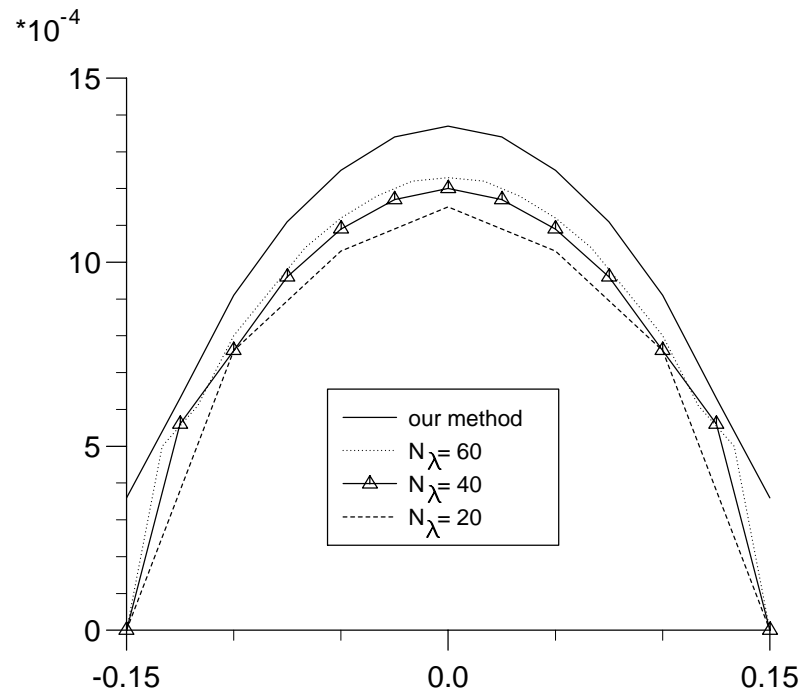

Figure 9: Comparison of the modulus of the current obtained from our method and from a method assuming the current intensity to vanish at both extremities of the wire. The parameters are the same as in Fig. 7, with $r=10^{-2} \lambda$. For our method, $N_{\lambda}=40$.

It is quite clear that the results at $r / \lambda=10^{-4}$ are very close to each other, in contrast with the results at $r / \lambda=10^{-2}$. Figure 9 shows the same results as Fig. 7 (with $r / \lambda=10^{-2}$ ) but the curves of current intensity have been drawn for increasing values of $N_{\lambda}$, from 20 to 60 , at least when the surface current is assumed to vanish at both extremities. The reader can control in Fig. 5 that the results obtained with our method (i.e. no assumption about the value of the current at the extremities) and repeated by the solid line in Fig. 9 are much more stable than the ones obtained with the usual approximation. It is worth noting that the results obtained from the classical hypothesis become closer and closer from our results as $N_{\lambda}$ is increased. Unfortunately, numerical instabilities on both sides of the curve begin to appear if $N_{\lambda}$ exceeds the value of 60 but Fig. 9 clearly shows that our method converges much more rapidly and is able to provide accurate results for moderate values of $N_{\lambda}$. It can be concluded that the assumption of a current intensity which vanishes at extremities may have catastrophic consequences on the numerical results for current intensity on the rest of the wire or for scattered field.

In our numerical implementation, we did not assume that the current intensity was conserved at a junction 
between wires.

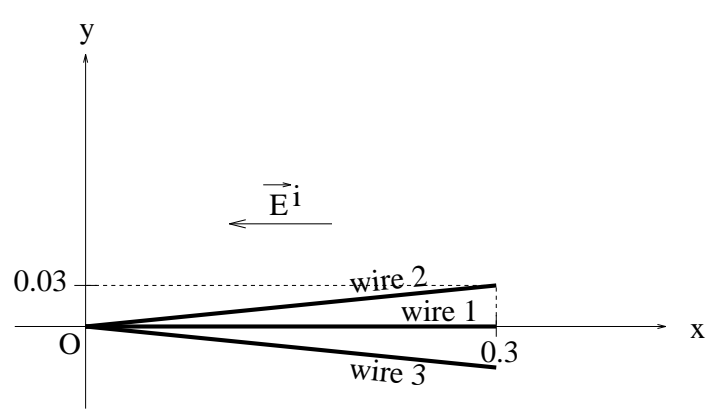

Figure 10: Scheme of a system of three connected wires with a junction making a sharp end.

We consider in Fig. 10 a system of three wires of length $0.3 \lambda$ and radius $10^{-2} \lambda$ lying in the $x y$ plane and connected in $O$. Each wire is very close to the $\mathrm{x}$-axis, the incident plane wave propagating in the $y$ direction. We have taken the incident electric field parallel to the $\mathrm{x}$-axis, thus charges should accumulate at point $O$. Figure 11 gives the intensity on the wires. It is to be noticed that the modulus of the sum of the algebraic intensities at the junction has been found to be equal to $0.4 \cdot 10^{-3}$ which is not negligible compared to the modulus of the intensity on each wire at the same point, for instance about $2.10^{-3}$ for wires 2 and 3 .

\section{Numerical study of three- dimensional metallic photonic crystal}

In contrast with two-dimensional crystals which are made with parallel wires, three-dimensional crystals exhibit interconnected wires in the three dimensions. The aim of this section is to investigate the properties of these more complicated structures and to compare them to the properties of two-dimensional crystals. The computation for $3 \mathrm{D}$ crystals is done with our code based on the numerical method described in section 4 . We are dealing with the three-dimensional crystal depicted in Fig. 12.

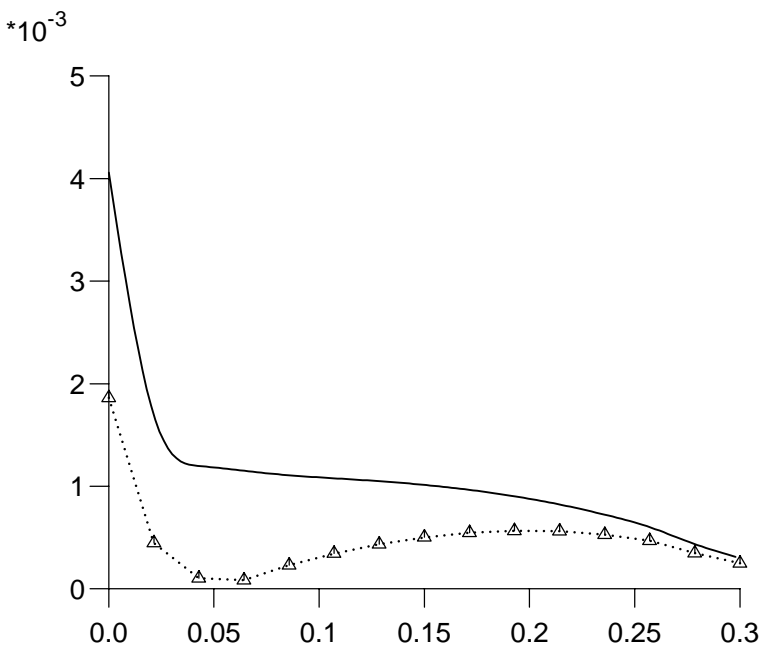

Figure 11: Modulus of the current along the three wires of Fig. 10, from the junction to the extremity (solid curve: wire 1, dashed curve: wires 2 and 3). Intensity on wire 2 has been found to be the same as intensity on wire 3 . The sum of the algebraic intensities at the junction $(x=0)$ is equal to $0.4 \cdot 10^{-3}$, thus not negligible compared to the intensity on each wire.

The crystal has a cubic geometry, with a period equal to unity in the $x, y$ and $z$ directions. The radius of the wires is equal to $10^{-2}$.

\subsection{Energy maps inside the crystal}

In order to illustrate qualitatively the main property of metallic photonic crystals, we have drawn maps of the electric energy $|\vec{E}|^{2}$ inside the crystal for two values of the wavelength, the first one $(\lambda=1.25)$ outside the gap, the second one $(\lambda=10)$ inside. The incident electric field is perpendicular to the figure (parallel to the $x$-axis). For each wavelength, the results obtained for the 3D model have been compared with those obtained from two simplified models:

- the simplified 3D model (S3D) where all the wires of the 3D crystal have been removed, except those which are parallel to the incident electric field. Thus, this model replaces the 3D crystal by a set of parallel wires of finite length. 


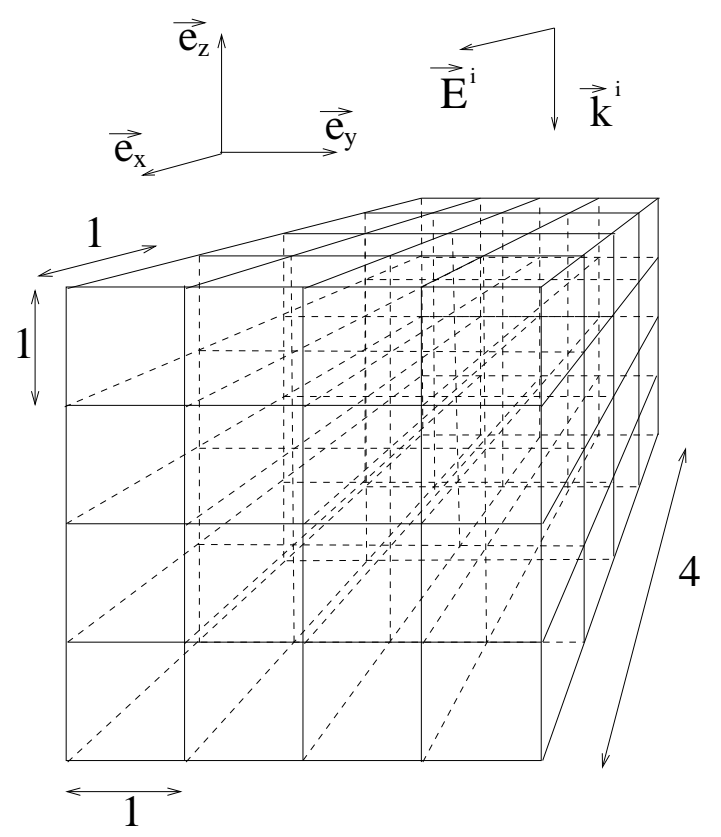

Figure 12: Three dimensional metallic cubic crystal centered at the origin and having $4 \times 4 \times 4=64$ elementary cells.

- the 2D model, where the wires of the S3D model are assumed to have infinite length.

The S3D model has needed the use of the same computer code as the 3D model, but the computation times is much smaller since the total length of the set of wires is reduced by a factor of 3 . On the other hand, the $2 \mathrm{D}$ model is much simpler to handle, and we have used a computer code devoted to 2D photonic crystals based on a rigorous theory of scattering [19]. Using a desktop workstation, a calculation at $\lambda=10$ needed computation times of the order of 30 minutes for the 3D model, 3 minutes for the S3D model and about one second for the 2D model. In fact, it emerges that the computation time required by the use of the 3D model is almost entirely devoted to the computation of the integrals in eqs. (8) and (9). Obviously, many of these integrals are identical, due to the periodicity of the structure. Taking this remark into account, we have been able to reduce the computation time to about 20 seconds for the 3D model and to 3 seconds for the S3D model.

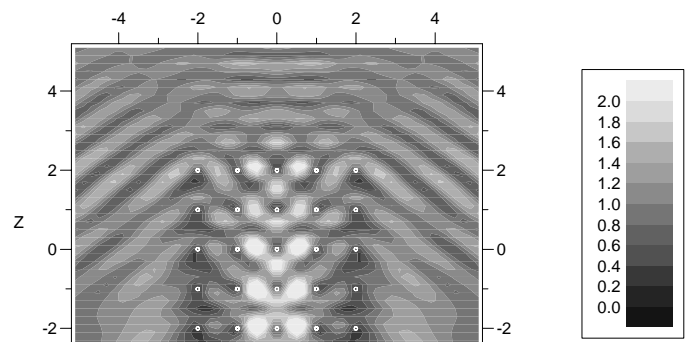

(a)

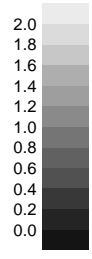

(b)

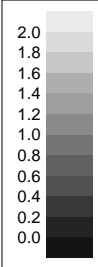

(c)

Figure 13: Energy map for $\lambda=1.25$ for the crystal of Fig. 12 with the three models: the 3D (a), S3D (b) and 2D (c) models. Polarization and direction of the plane wave are provided by Fig. 12. Light propagates through the structure and the maps are very similar. 

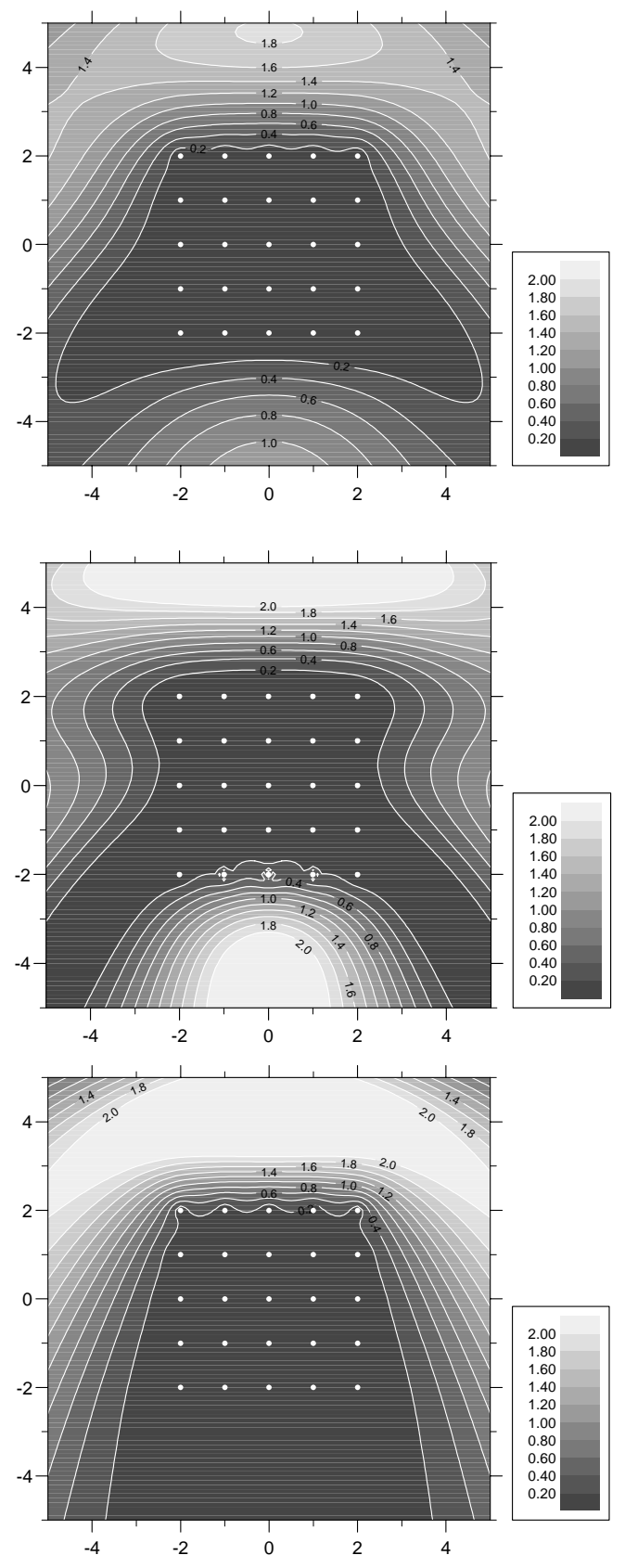

Figure 14: Same as Fig. 13 but for a wavelength $\lambda=$ 10 , i.e. inside the band gap.

Figure 13 (a) shows the map of $|\vec{E}|^{2}$ in the plane $x=$ 0.05 close to the symmetry plane $y z$ of the crystal (this small shift allows us to avoid the wires placed in the symmetry plane, where the electric field vanishes). The maps of Fig. 13 (b) and 13 (c) give the same results for the S3D and 2D models. At $\lambda=1.25$, the three models give very close results: light propagates through the structure. On the other hand, Fig. 14 shows that the field does not penetrate the crystal at $\lambda=10$. The results provided by the three methods remain very close for the field inside the crystal.

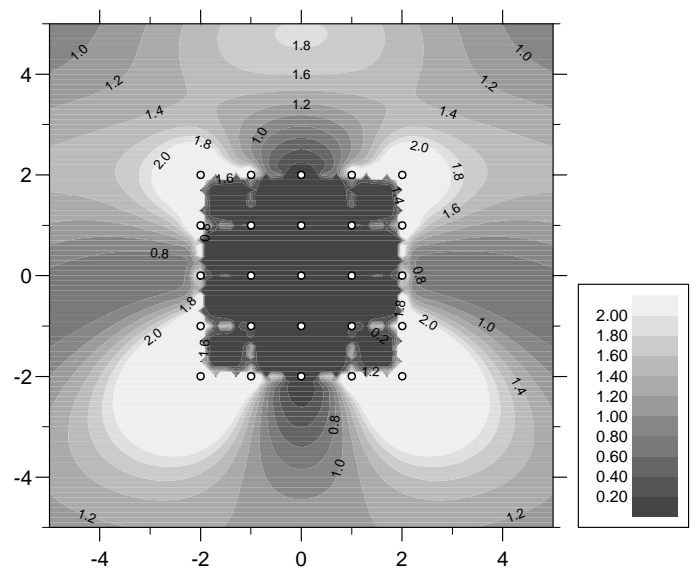

Figure 15: Same as Fig. 14 but for a different polarization: the electric field is parallel to the $y$-axis. For the S3D and 2D models, the incident field is perpendicular to the wires, thus the diffraction is negligible and the field modulus is constant. Hence the maps are not given.

Figure 15 gives an explanation. In this figure, the parameters remain the same as in Fig. 14, but the polarization of the incident light has changed: the incident electric field has been rotated by $90^{\circ}$, in such a way that it becomes orthogonal to the wires in the S3D and 2D models. As a consequence, the diffraction of the field by the wires is very small, i. e. the crystal is transparent and the field can penetrate inside it. On the other hand, in the 3D model, the electric field is parallel to another set of wires and nothing is changed. This property clearly shows the advantages of 3D crystals: they can control the propagation of light in any direction and any polarization. Nevertheless, a very interesting conclusion can be drawn from this section: the 2D model, which is much simpler than the 3D model, is able to provide accurate predictions on the behaviour of 3D metallic photonic crystals, provided the incident electric field is parallel to an edge of the elementary cubic cell. It will be shown in the next subsection that the gap of $3 \mathrm{D}$ crystal is almost independent of the direction of 
polarization of the incident light, thus that the use of the $2 \mathrm{D}$ model when the above condition is satisfied gives reliable predictions on the gaps of $3 \mathrm{D}$ crystals whatever the polarization will be. This is all the more interesting since numerous investigations have been made on 2D metallic photonic crystals. In particular, it has been shown from homogenization processes that 2D crystals can simulate a homogeneous material with a plasmon frequency in the microwaves region, which can be predicted accurately from a closed form formula [21]. It can be conjectured that the same formula can give the plasmon frequency of 3D crystals with a good precision. This result confirms the conjecture of specialists of Solid State Physics on the behaviour of 3D metallic photonic crystals [20].

\subsection{Photonic band gaps}

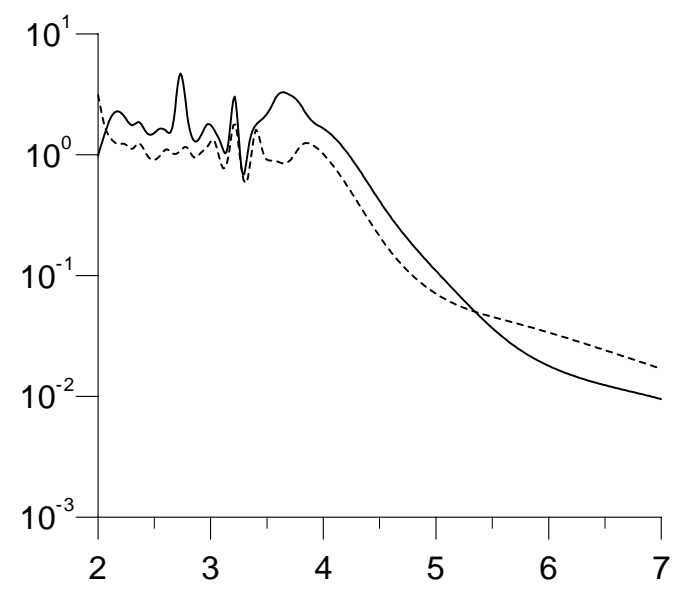

Figure 16: Mean value of the energy inside the crystal vs. wavelength. The average is taken over 64 points near the middle of the crystal. Solid curve: the incident wave is described in Fig. 12. Dashed curve: the wavevector is arbitrary chosen (see text).

The penetration of light inside the 3D crystal is estimated by computing the mean value of the squared modulus of the electric field over 64 points located near the middle of the structure. The solid curve in Fig.16 shows this mean value versus the wavelength when the incident electric field is parallel to one edge of the crystal, the incident wavevector being parallel to another edge (see Fig. 12). At wavelengths greater than 5, propagation inside the crystal becomes impossible. With the same incident wavevector, a rotation of the incident electric field would not change the gap. Indeed, an arbitrary polarization can be decomposed into two orthogonal polarization vectors parallel to the wires. On the other hand, it is not obvious that an arbitrary incident wavevector produces the same gap. The dashed curve in Fig. 16 shows that the cut-off wavelength remains nearly the same when the incident wave vector $\vec{k}_{i}$ and the incident electric field are respectively parallel to the vectors $\vec{u}$ and $\vec{v}$ given by:

$$
\begin{aligned}
& \vec{u}=\vec{e}_{x}+\vec{e}_{y}+\vec{e}_{z} \\
& \vec{v}=\vec{e}_{x}+\vec{e}_{y}-2 \vec{e}_{z}
\end{aligned}
$$

$\vec{e}_{x}, \vec{e}_{y}$ and $\vec{e}_{z}$ being the unit vectors of the axes.

Thus, the transmission gap remains nearly independent of the polarization and wavevector of the incident wave, a feature which makes $3 \mathrm{D}$ crystals more interesting than $2 \mathrm{D}$ crystals. Of course, this property could make it interesting to use 3D metallic photonic crystals for antennas, waveguides or cavities design.

\subsection{Gaps of doped crystals}

In order to get a doped crystal, three connected wires have been removed from the middle part of the 3D crystal shown in Fig. 12. Figure 17 shows the mean value of $|\vec{E}|^{2}$, computed as in subsection 6.2 , the incident wave being given by Fig. 12 (incident wave vector and polarization parallel to the edges of the crystal). A peak at $\lambda=4.5$ appears in the gap of the doped crystal, close to the edge of the gap. This peak is caused by the existence of a resonant mode of the cavity made inside the crystal by removing the three wires. It is worth noticing that this peak is not very narrow. This fact can be explained easily. The photonic crystal is small since it contains only $4 \times 4 \times 4$ elementary cells. Thus, the cavity inside the crystal have important losses and the resonance cannot be very sharp. Unfortunately, time computation and memory storage do not allow us to perform calculations for larger crystals. Nevertheless, Fig. 17 shows that cavities inside a metallic crystal exhibit the same effect as for dielectric crystals: peaks may appear inside the gap. 


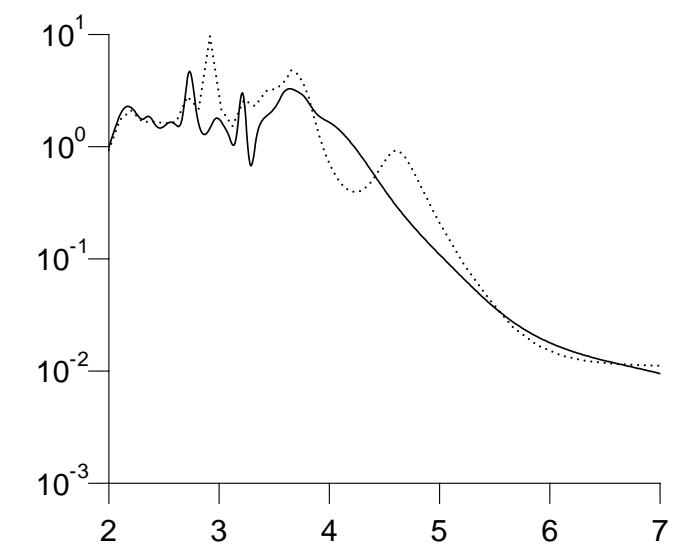

Figure 17: Dotted curve: same as Fig. 16 but for a doped crystal obtained by removing 3 connected wires in the middle of the crystal; solid curve: same as Fig. 16. The resonant excitation of a mode of the cavity induces a peak in the transmission at wavelength $\lambda=4.5$.

\section{Conclusion}

In the first part of the paper, an adaptation of the theory of scattering by thin wires developed by Harrington has been presented. The originality of this adaptation lies on two points: we do not assume any more that the current vanishes at free extremities of wires and we do not use Kirchhoff's law at junctions between wires. Thanks to this adaptation, we are able to deal with wires with lengths smaller than half a wavelength and moreover, the numerical implementation is simpler than that of the classical method. Using our computer code, we have been able to investigate the properties of $3 \mathrm{D}$ metallic photonic crystals and to compare these properties with those of $2 \mathrm{D}$ crystals. Even though the $3 \mathrm{D}$ crystal is the only one which presents gaps nearly independent of the wavevector and polarization of the incident wave, it emerges that, fortunately, the $2 \mathrm{D}$ model allows one to investigate with a reasonable accuracy the main properties of 3D crystals, specially the cut-off wavelength of the transmission gap. This is all the more interesting since the numerical study of 2D metallic crystals is much simpler and since formulae in closed form are able to predict the location of the gap with a good pre- cision [21].

The main problem encountered in our study of 3D crystals is the computation requirements of our code in memory storage. We are trying to overcome this problem by using models of crystals periodic (i.e. of infinite extend) in one or two directions. The periodicity of the problem in these directions can be used in order to increase the size of the crystal in the other ones. Finally, we are also trying to generalize to $3 \mathrm{D}$ crystals the mathematical homogenization process already achieved for $2 \mathrm{D}$ crystals.

\section{Acknowledgments}

The work described in this paper has been made in the course of a contract between the Laboratoire d'Optique Electromagnétique and the Direction des recherches, Ètudes et Techniques (French Ministery of Defence)

\section{References}

[1] E. Yablonovitch. "Inhibited spontaneous emission in solid-state physics and electronics". Phys. Rev. Lett., 58, 2059-2062, (1987).

[2] S. John. "Strong localization of photons in certain disordered dielectric superlattices". Phys. Rev. Lett., 58, 2486-2489, (1987).

[3] J. Joannopoulos, R. Meade, and J. Winn. Photonic Crystals. Princeton University Press, (1995).

[4] J. Rarity and C. Weisbuch, ed. Microcavities and Photonic Bandgaps: Physics and Applications, Vol. 324 of serie E, Dordrecht, (1996). NATO Advanced Study Institute, Kluwer Academic Publishers.

[5] C. Soukoulis. Photonic Band Gaps and Localization. Plenum, New York, (1993).

[6] Special Issue on Photonic Band Structures, Vol. 41. J. Modern Optics, (1994).

[7] Special Issue: Development and Applications of Materials Exhibiting Photonic Band Gaps, Vol. 10. J. Opt. Soc. Am. B, (1993).

[8] D. Maystre. "Electromagnetic study of photonic band gaps". Pure Applied Optics, 3, 975-993, (1994). 
[9] G. Tayeb and D. Maystre. "Rigorous theoretical study of finite size two-dimensional photonic crystals doped by microcavities". J. Opt. Soc. Am. A, 14, 3323-3332, (1997).

[10] E. Ozbay, B. Temelkuran, M. Sigalas, G. Tuttle, C. Soukoulis, and K. Ho. "Defect structures in metallic photonic crystals". Applied Physics Letter, 69, 3797-3799, (1996).

[11] S. Cheng, R. Biswas, E. Ozbay, J. McCalmont, G. Tuttle, and K. Ho. "Optimized dipole antennas on photonic band gap crystal". Applied Physics Letter, 67, 3399-3401, (1995).

[12] E. Brown and O. McMahon. "High zenithal directivity from a dipole antenna on a photonic crystal". Applied Physics Letter, 68, 1300-1302, (1996).

[13] C. Maggiore, A. Clogston, G.Spalek, W. Sailor, and F. Mueller. "Low-loss microwave cavity using layered-dielectric materials". Applied Physics Letter, 64, 1451-1453, (1994).

[14] D. Smith, S. Schultz, N. Kroll, M. Sigalas, K. Ho, and C. Soukoulis. "Experimental and theoretical results for a two-dimensional metal photonic band-gap cavity". Applied Physics Letter, 65, 645-647, (1994).

[15] E. Brown and O. McMahon. "Large electromagnetic stop bands in metallodielectric photonic crystal". Applied Physics Letter, 67, 2138-2140, (1995).

[16] D. F. Sievenpiper, M. E. Sickmiller, and E. Yablonovitch. "3D wire mesh photonic crystals". Phys. Rev. Lett., 76(14), 2480-2483, (1996).

[17] R. Harrington. "Matrix methods for field problems". Proc. IEEE, 55(2), 136-149, (1967).

[18] E. Miller and F. Deadrick. "Some computational aspect of thin-wire modeling". In Topics in Applied Physics,R. Mittra, ed., Vol. 3, chapter 4, page 89. (Springer-Verlag Berlin Heidelberg New York, 1975).

[19] D. Felbacq, G. Tayeb, and D. Maystre. "Scattering by a random set of parallel cylinders". J. Opt. Soc. Am. A, 11, 2526-2538, (1994).
[20] J. B. Pendry. "Calculating photonic band structure". J. Phys.: Condens. Matter, 8, 1085-1108, (1996).

[21] G. Guida, D. Maystre, G. Tayeb, and P. Vincent. "homogenization of two-dimensional metallic photonic crystals". submitted to the J. Opt. Soc. Am. B, ( ), , (1997).

[22] G. Thiele. Computer Techniques for Electromagnetics, chapter Wire antennas, pages 7-96. Pergamon Press Ltd., Oxford, (1973).

[23] J. L. Roumiguières. Personal communications.

[24] J. Berntsen, T. Espelid, and A. Genz. "An adaptative algorithm for the approximate calculation of multiple integrals". ACM Transactions on Mathematical Software, 17, 437, (1991).

[25] J. Berntsen, T. Espelid, and A. Genz. "Algorithm 698: DCUHRE: an adaptative multidimensional integration routine for a vector of integrals". ACM Transactions on Mathematical Software, 17, 452, (1991).

[26] M. Saillard, P. Vincent, and D. Maystre. "A finite element method for electromagnetic subsurface tomography". In Finite element software for microwave engineering, T. Itoh, G. Pelosi, and P. Silvester, ed., chapter 11. (Wiley-Interscience, 1996).

[27] M. Born and E. Wolf. Principles of optics. Pergamon press, (1980). 